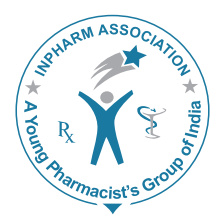

\title{
Investigation of various practical techniques to enhance dissolution of ezetimibe from oral tablets: A comparative study
}

\author{
Prema Kumari Nanam¹, Kiran Thadkala', Chinta Sailu², Jithan Aukunuru \\ ${ }^{1}$ Mother Teresa College of Pharmacy, Osmania University, ${ }^{2}$ University College of Technology, \\ Osmania University, Hyderabad, Andhra Pradesh, India
}

\begin{abstract}
Objective: The aim of this work was to investigate and compare various practical techniques to enhance dissolution of ezetimibe, a Class II Biopharmaceutics Classification System compound. Ezetimibe is a poorly soluble compound with variable oral bioavailability. Nanosuspensions, hydroxypropyl-beta-cyclodextrin (HP $\beta C D$ ) complexes as well as combination of surfactant were techniques investigated. Materials and Methods: Nanosuspension of ezetimibe was prepared using solvent-antisolvent precipitation technique. The nanosuspensions were characterized by powder $X$-ray diffractometry and scanning electron microscopy. HP $\beta C D$ inclusion complexes were prepared using physical mixture, co-evaporation, and kneading methods. These complexes were characterized using Fourier transform infrared spectroscopy (FTIR) and differential scanning calorimetry (DSC). Ezetimibe tablet formulations containing drug nanosuspensions, HP $\beta C D$ complexes as well as sodium lauryl sulfate (SLS) enrichment were prepared and evaluated for dissolution. Results and Discussion: Nanosuspensions of ezetimibe with a mean particle size of $900 \mathrm{~nm}$ were successfully prepared using solvent-antisolvent precipitation. FTIR and DSC studies confirmed the formation of ezetimibe inclusion complexes. Tablets prepared using pure drug showed very poor dissolution. In contrast, tablets of nanosuspensions, HPBCD inclusion complexes and SLS mixtures showed enhanced dissolution. The tablets prepared using nanosuspensions demonstrated enhanced dissolution rate when compared to the formulations prepared using HP $\beta C D$ complexes and SLS enrichment. All the techniques investigated can be used to enhance the dissolution of ezetimibe and thus can enhance the oral bioavailability and also reduce the fluctuations in the oral bioavailability. Conclusion: The techniques that were employed in this present work, nanosuspensions seem to be better when compared to cyclodextrin inclusion complexes and SLS enrichment in enhancing solubility and dissolution rate of ezetimibe.
\end{abstract}

Key words: Drug-sodium lauryl sulphate tablets, ezetimibe, hydroxypropyl-beta-cyclodextrin complexes, nanosuspension, nanosuspension tablets, sodium lauryl sulphate

\begin{tabular}{|l|l|}
\hline \multicolumn{2}{|c|}{ Access this article online } \\
\hline \multirow{2}{*}{ Journal Sponsor } & \multicolumn{1}{|l|}{ Website: } \\
www.jyoungpharm.org \\
\hline \multirow{2}{*}{ www.phcog net } & DOI: \\
\cline { 2 - 2 } & $10.5530 /$ /jyp.2014.1.2 \\
\hline
\end{tabular}

\section{INTRODUCTION}

Oral drug delivery is the most common method for administration of drugs. ${ }^{1}$ For the assessment of new drug entities, researchers and industry invariably use oral route in the exploratory stages. In the current situation, a large number of drugs reaching the hands of formulators are

*Address for correspondence:

Mr. Jithan Aukunuru, Mother Teresa College of Pharmacy, Osmania University, Hyderabad, Andhra Pradesh,

India.E-mail:aukunjv@gmail.com 
poorly water soluble and thus their oral pharmacokinetics are compromised with the oral bioavailability and absorption across the gastrointestinal tract (GIT) is either low or variable. ${ }^{2}$ Different techniques have been successfully investigated to improve the solubility. These techniques modify the physicochemical properties of the drug or add novel functionality to the molecular structure of the drug or develop newer formulations. Such techniques include microparticles, nanoparticles, liposomes, nanosuspension, cyclodextrin inclusion complexes, surfactant-enrichment, salts, $\mathrm{pH}$ modification, prodrugs, nanoemulsions and redispersable emulsion powders. ${ }^{3,4}$ Of these many techniques, practical and clinical reality has been achieved with nanosuspensions, cyclodextrin inclusion complexes and surfactant enrichment. The enhancement in dissolution rate of a drug enhances the oral bioavailability as well as reduces the fluctuations in the bioavailability of poorly soluble compounds.

Ezetimibe is a lipid-lowering compound that selectively inhibits the absorption of cholesterol and related phytosterols from the intestine. ${ }^{5}$ It is a Biopharmaceutics Classification System Class II drug with low solubility and high permeability. Water solubility of ezetimibe is 8.46e-03 $\mu \mathrm{g} / \mathrm{l}$. It is a weakly basic compound and thus contains ionizable groups. Its $\log P=4$.5. Its strongest acidic pKa and strongest basic pKa are 9.48 and -3 , respectively. As it is poorly soluble in water, its oral absorption is less, and it was found that it also has the problem of variable pharmacokinetics after oral administration. ${ }^{6}$ This could be mainly attributed to poor solubility and poor dissolution rate. Its absolute bioavailability cannot be determined as it has very low solubility that an intravenous injection cannot be prepared. Even though ezetimibe contain ionizable groups, it shows essential $\mathrm{pH}$ independent solubility characteristics across GIT $\mathrm{pH}$ range. ${ }^{7}$ Thus, $\mathrm{pH}$-based strategies to improve solubility/dissolution characteristics (e.g. salts, addition of $\mathrm{pH}$ modifiers) were not the first line strategy. The other strategies can be attempted with success, but their manufacture is not practical. In this regard, nanosuspensions, cyclodextrin complexes and addition of surfactants may be the practical approaches. Thus, the objective of this study was to enhance the dissolution rate of ezetimibe so as to solve its poor and variable bioavailability problems using selected techniques, which include nanosuspensions, hydroxypropyl-betacyclodextrin $(\mathrm{HP} \beta \mathrm{CD})$ complexes and sodium lauryl sulfate (SLS) enrichment and compare each of these techniques. The techniques selected are practical and the formulations prepared using these techniques can be conveniently commercialized.

\section{MATERIALS AND METHODS}

Ezetimibe and $\mathrm{HP} \beta C D$ were obtained as a gift sample from Suven Nishtaa Ltd., Hyderabad, India. Ethanol, Tween 80 and SLS were obtained from SD fine chemicals limited. Magnesium stearate, lactose, croscarmellose sodium and povidone K 30 were obtained from Lab tech. All the other ingredients were used of analytical grade. All the equipment used in the study is routinely calibrated using procedures described by the manufacturers.

\section{Preparation and characterization of ezetimibe nanosuspensions}

Ezetimibe is a lipid lowering agent. PUMchem indicates its molecular formula as $\mathrm{C}_{24} \mathrm{H}_{21} \mathrm{~F}_{2} \mathrm{NO}_{3}$ and molecular weight as 409.42. Ezetimibe nanosuspensions were prepared by a modification of solvent-antisolvent precipitation method as previously described for other drugs. ${ }^{8}$ Ethanol was used as solvent and water was used as antisolvent. Tween 80 was used as surfactant to stabilize the nanosuspension. The drug ezetimibe $(100 \mathrm{mg})$ was dissolved in solvent ethanol $(4 \mathrm{ml})$. A $0.2 \mathrm{ml}$ of tween 80 was dissolved in distilled water $(20 \mathrm{ml})$. Ethanolic solution was injected drop wise into tween 80 solution with a constant stirring on a magnetic stirrer. Ethanol was evaporated under vaccum to precipitate the nanosuspension. The product was recovered by centrifugation at $7000 \mathrm{rpm}$ for 10 min using a Remi high speed centrifuge, washed twice with water and then freeze dried. The freeze dryer used was Delvac Mini Lyodel, Chennai, India. Freeze dried nanosuspension was then used for further studies. The yield of the formulation from one batch was determined using an assay method developed using ultra violet-vis. spectrophotometer. Briefly, $10 \mathrm{mg}$ of ezetimibe nanosuspensions were dissolved in $10 \mathrm{ml}$ of methanol, the aliquot was centrifuged and the supernatant was taken for the drug assay. The $\lambda_{\text {max }}$ used for the assay was $232 \mathrm{~nm}$. The percentage yield was calculated using the formula:

$\%$ Yield $=\frac{\text { Amount of the drug in nanususpensions }}{\text { Amount of the drug taken for preparation }} \times 100$

The mean particle size was determined using an optical microscope. In order to examine the particle surface morphology and shape, scanning electron microscopy (SEM) was used. A concentrated aqueous suspension was spread over a slab and dried under vacuum. The sample was shadowed in a cathodic evaporator with gold layer $20 \mathrm{~nm}$ thick. Photographs were taken using a JSM-5200 SEM (Tokyo, Japan) operated at $20 \mathrm{kV}$. The crystallinity of the drug was evaluated using X-ray powder diffraction (XRPD). X-ray spectra were recorded with 
X'Pert-PRO multipurpose X-ray diffractometer (PANalytical, Tokyo, Japan) using Ni-filtered, $\mathrm{Cu}$ Ka radiation, a voltage of $45 \mathrm{kV}$, and a current of $40 \mathrm{~mA}$ with a scintillation counter. The instrument was operated in the continuous scanning speed of $10^{\circ} /$ min over a $2 \theta$ range of $10^{\circ}$ to $80^{\circ}$.

Preparation and characterization of hydroxypropyl-beta-cyclodextrin inclusion complexes of ezetimibe

Ezetimibe HPßCD inclusion complexes were prepared using three methods as described earlier. ${ }^{9,10}$ These methods are physical mixing, kneading method, and co-evaporation method. Preliminary phase solubility experiments were conducted to determine drug: HPBCD ratio for optimum binding. Based on the results, 1:1 weight ratio was considered optimum and we used this ratio in all the studies. In physical mixing method, ezetimibe and $\mathrm{HPBCD}$ were accurately weighed in equal ratios of 1:1 and were prepared by simply mixing powders with a spatula for $15 \mathrm{~min}$ and then sieved through $120 \#$ to obtain final desired inclusion complex. In case of kneading method, 1:1 ratio of drug and HPBCD were weighed. HPBCD and distilled water mixed together in a mortar so as to obtain a homogenous paste. Drug was added slowly; while mixing, a small quantity of methanol was added to assist the dissolution of drug. Mixtures were then blended for $2 \mathrm{~h}$ using an appropriate quantity of water to maintain suitable consistency. The paste was dried in oven at $45-50^{\circ} \mathrm{C}$ for $24 \mathrm{~h}$. The dried complex was pulverized and then sieved through $120 \#$ to obtain final desired inclusion complex. In co-evaporation, drug and $\mathrm{HPBCD}$ were accurately weighed in the equal ratios of $1: 1$ and dissolved in the same quantities $(5 \mathrm{ml})$ of methanol and water respectively. Both the solutions were mixed and solvents were evaporated by controlled heating at $45-50^{\circ} \mathrm{C}$. The resulting solid was pulverized and then sieved through 120 \# to obtain final desired particle size of inclusion complex. It was made sure that the entire methanol was removed from the complex at the end of its preparation.

Ezetimibe HPBCD inclusion complexes were characterized by Fourier transform infrared spectroscopy (FTIR) and DSC. The FTIR spectra of drug, cyclodextrin, physical mixture, kneading method, and co-evaporation method were obtained. About $5 \mathrm{mg}$ of sample was mixed thoroughly with $100 \mathrm{mg}$ potassium bromide IR powder and compacted under vacuum a pressure of about 12 Psi for $3 \mathrm{~min}$. The resulting disc was mounted in a suitable holder in Perkin Elmer IR spectrophotometer, and the IR spectrum was recorded from $4000 / \mathrm{cm}$ to $625 / \mathrm{cm}$ in a scan time of $12 \mathrm{~min}$. Thermal properties of the powder samples were investigated with a differential scanning calorimetry (DSC). DSC measurements were taken by the Perkin Elmer's PYRIS Diamond DSC. The temperature was calibrated with pure indium. All measurements were performed under a high-purity nitrogen atmosphere to minimize degradation. Approximately, $10 \mathrm{mg}$ of sample was analyzed in an open aluminum pan, and heated at scanning rate of $10^{\circ} \mathrm{C} / \mathrm{min}$ between $0^{\circ} \mathrm{C}$ and $400^{\circ} \mathrm{C}$. Magnesia was used as the standard reference material.

\section{Preparation of ezetimibe sodium lauryl sulfate powder complex}

The ezetimibe SLS complex were prepared by addition of SLS in different amounts $(0.1 \%, 0.2 \%$, and $1 \%)$ to the drug. The powder mixture was thoroughly blended and sieved to obtain powder of desired size. This technique is called surfactant enrichment, is commonly used in industry and has been previously described. ${ }^{11}$

\section{Preparation and evaluation of ezetimibe tablets using nanosuspensions, cyclodextrin complexes and sodium lauryl sulfate mixtures}

Tablets containing pure drug, nanosuspensions, cyclodextrin inclusion complexes, and SLS enrichment were prepared using conventional wet granulation technique. Compressed tablets each containing $10 \mathrm{mg}$ of ezetimibe were prepared by wet granulation method employing pure ezetimibe, nanosuspensions, cyclodextin complexes, surfactantenriched drug. Lactose was used as diluent to adjust the weight of the tablet to $200 \mathrm{mg}$, acacia $(2 \%)$, talc $(2 \%)$ and magnesium stearate $(2 \%)$ were incorporated respectively as a binder and lubricants. The tablet granules were compressed into tablets on a Cadmach 16-station rotary tablet punching machine (M/s Cadmach Engineering Co. Pvt. Ltd., Mumbai, India) using $9 \mathrm{~mm}$ concave punches. All the tablets prepared were evaluated for content of active ingredients, hardness, friability, disintegration time and dissolution rate as per official (IP) methods. Bulk density apparatus (Cintex industrial corporation, Mumbai, India), hardness tester (Monsanto), dissolution apparatus USP (Electrolab, TDT-08 L) were used. In-vitro dissolution studies of tablet formulations were carried out using USP apparatus II paddle method. Accurately, weighed tablets were added to $900 \mathrm{ml}$ of buffer media (acetate buffer $\mathrm{pH} 4.5)$ at $37^{\circ} \mathrm{C} \pm 0.5^{\circ} \mathrm{C}$ and stirred at $50 \mathrm{rpm}$. An aliquot of $10 \mathrm{ml}$ was withdrawn at different time intervals. An 
equal volume of fresh dissolution medium was immediately replaced. The samples were assayed spectrophotometrically at $232 \mathrm{~nm}$.

\section{Statistical analysis}

Results are expressed as mean \pm standard error of the mean of six samples per treatment group. Data were analyzed using a one-way analysis of variance followed by Dunnett test. Differences were considered as significant at $P \leq 0.05$.

\section{RESULTS AND DISCUSSION}

In this study, various practical techniques of enhancement of solubility of a poorly soluble compound ezetimibe were investigated and compared. The novel technique that was investigated to enhance the dissolution rate of a poorly soluble compound is nanosuspensions. Poor solubility, incomplete dissolution, and insufficient efficacy are the major problems of oral drug administration. Due to smaller particle size and much larger surface to volume ratio, oral nanosuspensions or nanocrystals are specially used to solve these problems. In case of azithromycin nanosuspensions, more than $65 \%$ drug was found to be dissolved in $5 \mathrm{~h}$ as compared with $20 \%$ of micronized drugs. ${ }^{12}$ By using the standard manufacturing techniques, drug nanosuspensions can be simply incorporated into various dosage forms. ${ }^{13}$ The other techniques that were investigated to enhance the solubility and dissolution rate of ezetimibe are use of cyclodextrins and surfactant enrichment, which are popular in pharmaceutical formulation development. ${ }^{10,11}$

Nanosuspension of ezetimibe was successfully prepared using the technique employed in this investigation. Particles were spherical and had an average size of $0.9 \mu \mathrm{m}$ with a yield of $80 \%$ (Figure 1). XRPD was used to investigate the physical nature of the drug in the nanosuspensions. From the XRPD graphs, it was observed that the crystallanity of the drug was changed in the nanosuspensions (Figure 2). The peaks obtained for pure drug was very clear and sharp and the intensity of the peaks was very high when compared with peaks of ezetimibe nanosuspensions. Reduction in the peak intensity indicates the change in crystal structure. From this, we can conclude that there was reduction in the crystallanity and change into amorphous structures upon fabricating into ezetimibe nanosuspensions. Nanosuspensions of ezetimibe were not previously investigated.

Inclusion complexes of ezetimibe with HPßCD were prepared successfully by physical mixture, co-evaporation and kneading method in a weight ratio of 1:1. This was confirmed by FTIR and DSC studies. The chemical interaction between the drug and the carrier can be observed by changes in the infrared (IR) profile of complexes and can be interpreted by careful study of the spectrum. Representative FTIR spectra are shown in Figure 3. The FTIR spectra's of the complexes were compared with spectrum of pure HPBCD and pure drug. When compared to pure drug, it has been observed that there is reduction in peak intensities of several functional groups in the complexes. Change in peak intensity indicates the complex formation. Therefore, it has been confirmed that inclusion complex of ezetimibe is formed by complexation with HPßCD. This was further corroborated by DSC studies. DSC is used to detect all processes in which energy is required or produced. The ezetimibe showed a melting peak at $184^{\circ} \mathrm{C}$ (Figure 4). Peak of ezetimibe at $184^{\circ} \mathrm{C}$ was present at the same position in all the samples with a reduction of intensity. Reduction in intensity is interpreted as formation of the complex. FTIR and DSC results suggest the trapping of drug in HPBCD

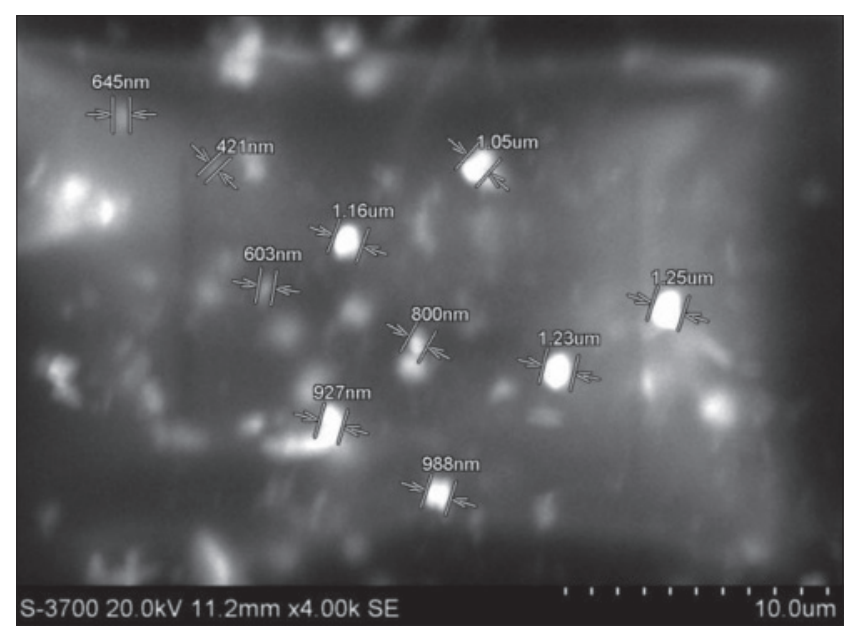

Figure 1: Scanning electron microscope pictures of nanosuspension

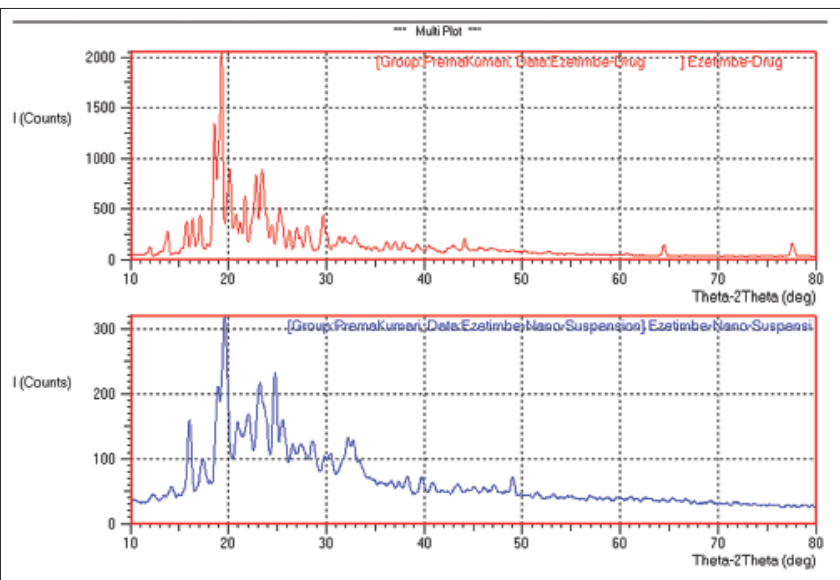

Figure 2: Powder X-ray diffraction of pure drug and nanosuspension 


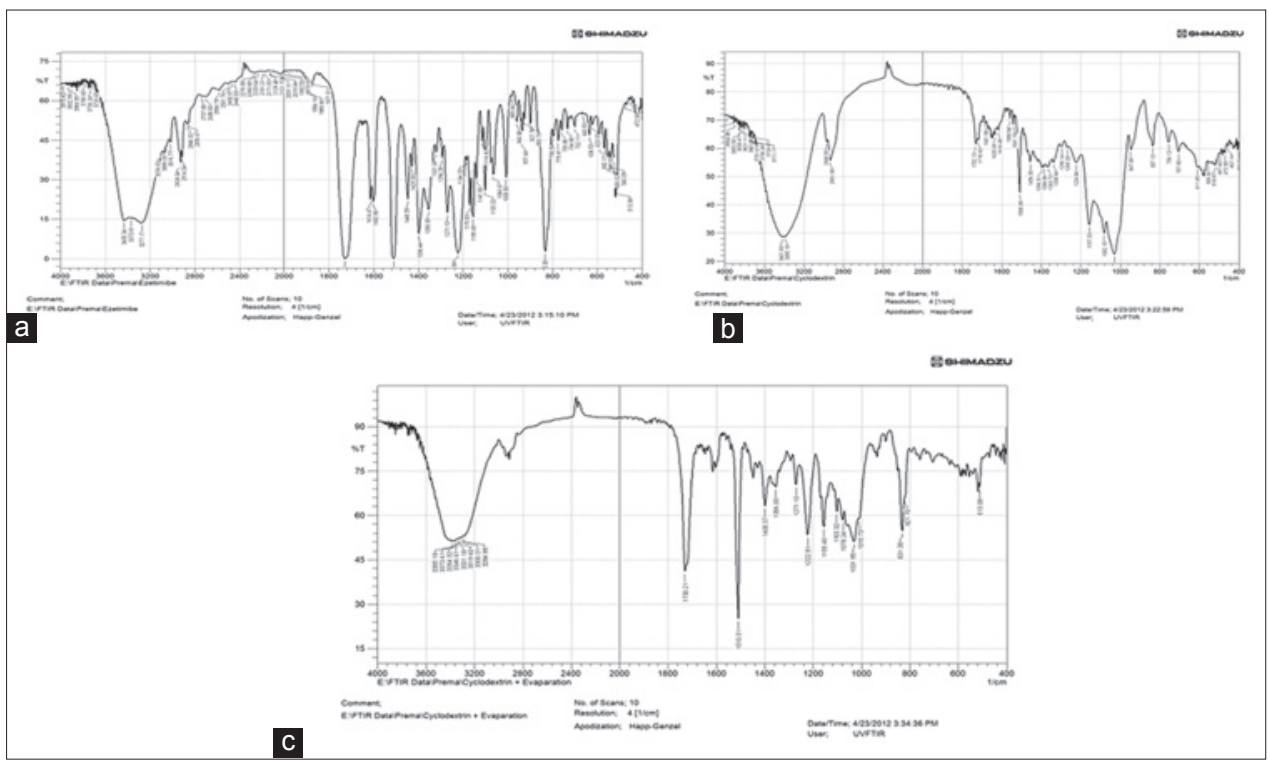

Figure 3: Fourier transform infrared spectroscopy graphs of cyclodextrin complexes. (a) Pure drug; (b) cyclodextrin; (c) cyclodextrin complexes prepared using co-evaporation

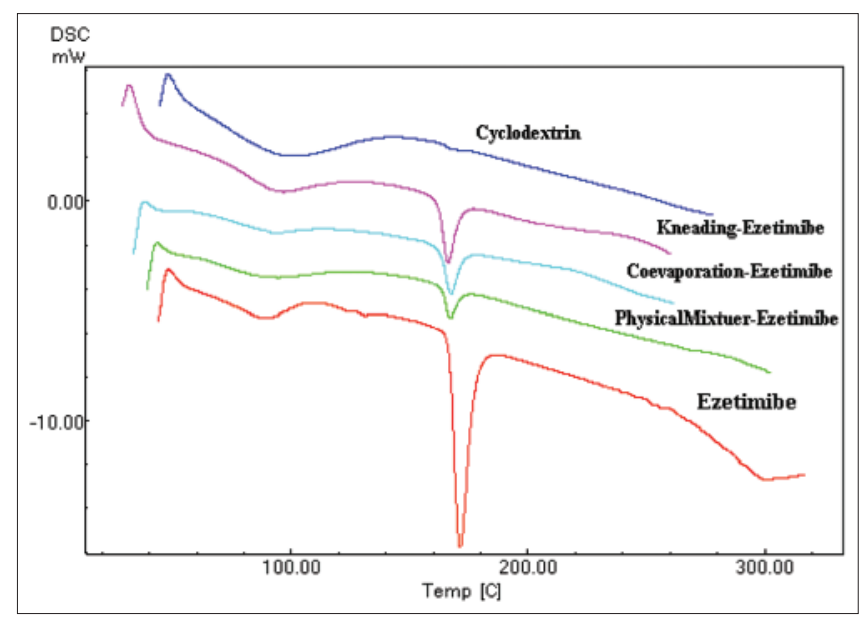

Figure 4: Cumulative differential scanning calorimetry graph of drugcyclodextrin complexes

in all the techniques. Such an interpretation was previously made in the literature. ${ }^{14}$ The enhancement of dissolution of ezetimibe with $\mathrm{HPBCD}$ was previously investigated by Taupitz et al. ${ }^{7}$ However, in their study, the aim was to improve the solubility of ezetimibe along with simvastatin in fixed dose combination formulations. HPßCD enhanced the dissolution rate of ezetimibe. We proved the results for ezetimibe tablets and not in combination with other drugs.

Ezetimibe tablets were prepared with the nanosuspensions, cyclodextrin inclusion complexes and surfactants using wet granulation technique. The prepared tablets were evaluated for the following parameters: weight variation, friability, hardness, disintegration time, assay, content uniformity, dissolution studies, and the results were compared. Tablet parameters indicated that good quality of tablets was

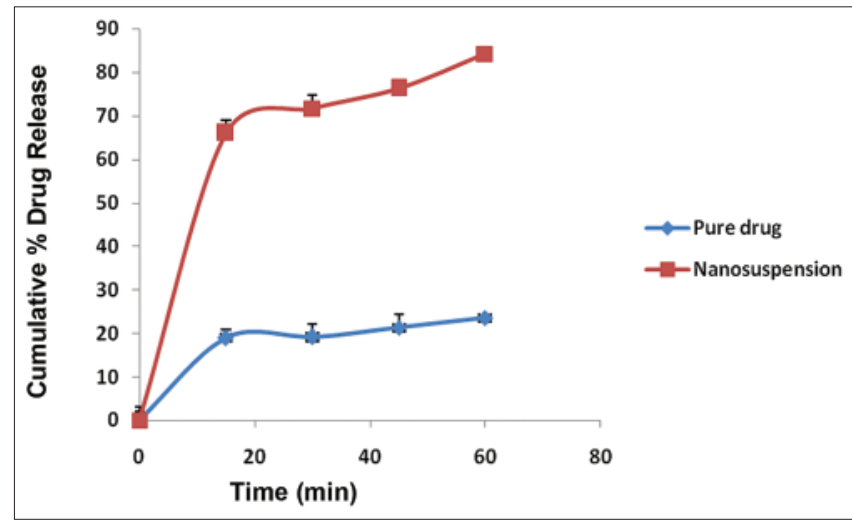

Figure 5: Cumulative \% drug release from ezetimibe tablets prepared using pure drug and nanosuspensions

Table 1: Evaluation of ezetimibe tablets prepared using various approaches

\begin{tabular}{lccc}
\hline $\begin{array}{l}\text { Evaluation } \\
\text { parameters }\end{array}$ & $\begin{array}{c}\text { Nanosuspension } \\
\text { tablets }\end{array}$ & $\begin{array}{c}\text { Cyclodextrin } \\
\text { complex } \\
\text { tablets }\end{array}$ & $\begin{array}{c}\text { Tablets } \\
\text { containing } \\
\text { surfactant }\end{array}$ \\
\hline Weight variation (\%) & $100 \pm 2$ & $100 \pm 2$ & $100 \pm 2$ \\
Hardness $\left(\mathrm{kg} / \mathrm{cm}^{2}\right)$ & 2.5 & 2.7 & 3.0 \\
Friability $(\%)$ & 1.8 & 1.9 & 1.4 \\
Disintegration (s) & 10 & 15 & 15 \\
Content & $100 \pm 0.5$ & $100 \pm 0.5$ & $100 \pm 0.5$ \\
uniformity (\%) & & & \\
\hline
\end{tabular}

prepared (Table 1). The results of dissolution studies demonstrated interesting results. The onset of dissolution of tablets prepared using unprocessed pure ezetimibe was very low $(20.56 \%$ in $1 \mathrm{~h})$ when compared with the tablets prepared using nanosuspensions (Figure 5). It was observed that there was an increase in $\%$ drug release $(82.4 \%$ in $1 \mathrm{~h}$ ) with tablets prepared using ezetimibe nanosuspensions.

Journal of Young Pharmacists Vol 6 • Issue 1 • Jan-Mar 2014 
It clearly indicates that nanosuspension formulation has been a successful technique to improve the dissolution rate thereby could enhance oral bioavailability and reduce fluctuations in oral bioavailability. Cyclodextrin complexes also resulted in enhancement of dissolution rate (Figure 6). Complexes prepared using co-evaporation and physical mixing significantly enhanced dissolution rates within $1 \mathrm{~h}$ compared with pure drug and complexes prepared using kneading technique. Inclusion complexes prepared using co-evaporation and physical mixing significantly enhanced dissolution rate of ezetimibe $(59.2 \%, 32.75 \%$ within $1 \mathrm{~h}$ ). Even dissolution rate of complexes prepared using kneading method also increased $(28.71 \%$ within $1 \mathrm{~h})$. The use of surfactants to improve the dissolution performance of poorly soluble drug products has also been successfully employed. There was an increase in dissolution as the concentration of surfactant increased (Figure 7). A 53.21\% of the drug was released in $1 \mathrm{~h}$ in the dissolution studies with the addition of $1 \%$ SLS. All the results demonstrated statistical significance.

Although oral bioavailability of ezetimibe was not measurable because of intravenous formulation developmental problems, the studies from administering oral formulations in humans indicated that the drug exhibited moderate intersubject variability with coefficient of variation ranging from $34-43 \%$ to $32-37 \%$ for $\mathrm{Cmax}$ and AUC, respectively. ${ }^{15}$ Several formulation approaches have been addressed to reduce this variability ${ }^{16-21}$ Such successful formulations include nanoemulsions, nanocrystals, self-nanoemulsifying granules, solid dispersions, etc. If these studies are carefully reviewed, it could indicate that nanotechnology can solve this problem of variable bioavailability of ezetimibe in a better way. ${ }^{16-20}$ Cyclodextrin inclusion complexes were also investigated to enhance the solubility and dissolution

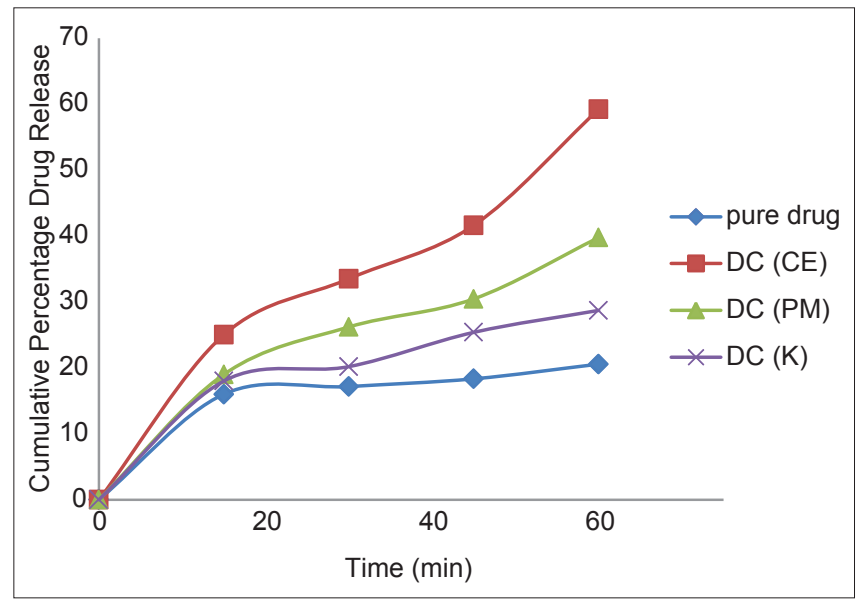

Figure 6: Cumulative \% drug release from ezetimibe tablets prepared using various drug-cyclodextrin complexes (DC) (PM: Physical mixture; CE: Coevaporation; K: Kneading) of ezetimibe and probably may reduce the fluctuations in its gastro-intestinal tract absorption. ${ }^{7}$ Since earlier studies have given us leads that nanotechnology can solve the problem to a better extent and since nanosuspensions of ezetimibe were not investigated, we set to investigate this approach in our study. From dissolution profiles from the tablets containing ezetimibe prepared using various techniques, nanosuspensions are found to enhance the dissolution rate to a greater extent compared with the other techniques. In this study, three different practical approaches for reduction in the variability of gastrointestingal tract absorption of ezetimibe have been investigated, which include nanosuspensions, cyclodextrin inclusion complexes and sodium lauryl sulfate enriched formulations. All the techniques demonstrated an increase in dissolution of the drug and could therefore reduce the fluctuations in the oral bioavailability of ezetimibe. The technology can be commercialized to obtain better ezetimibe formulations.

\section{CONCLUSIONS}

Taken together, results of this work indicate that all the practical techniques employed here led to an improvement in dissolution of ezetimibe and results are comparable to similar studies conducted earlier with other techniques. Within the techniques that were employed in this research work, nanosuspensions seem to be better when compared to cyclodextrin inclusion complexes and SLS enrichment in enhancing solubility and dissolution rate of ezetimibe.

\section{AUTHOR CONTRIBUTION}

This work is a part of M. Pharm project of Prema Kumari Nannam and a part of Ph.D. project of Kiran Thadkala. Prema Kumari and Kiran have conducted the experiments.

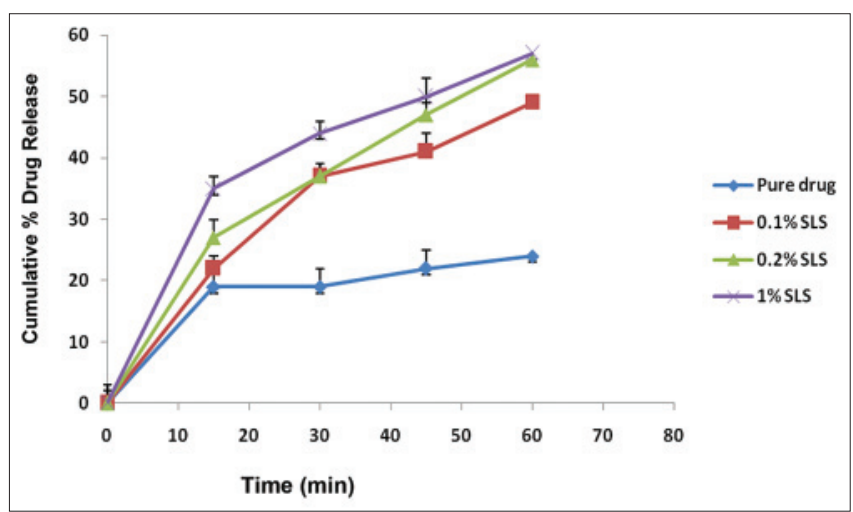

Figure 7: Cumulative \% drug release from ezetimibe tablets prepared using various amounts of sodium lauryl sulphate 
The experiments were designed by both Dr. Chinta Sailu and Dr. Jithan Aukunuru. The manuscript was written by Dr. Jithan Aukunuru and for this editorial assistance was provided by Prema Kumari and Kiran.

\section{REFERENCES}

1. Aukunuru J. Oral Drug Delivery Technology. 2nd ed. Hyderabad, India: Pharma Book Syndicate; 2012.

2. Stegemann S, Leveiller F, Franchi D, de Jong H, Lindén H. When poor solubility becomes an issue: from early stage to proof of concept. Eur J Pharm Sci 2007;31:249-61.

3. Savjani KT, Gajjar AK, Savjani JK. Drug solubility: importance and enhancement techniques. ISRN Pharm 2012;2012:195727.

4. Kotta S, Khan AW, Pramod K, Ansari SH, Sharma RK, Ali J. Exploring oral nanoemulsions for bioavailability enhancement of poorly water-soluble drugs. Expert Opin Drug Deliv 2012;9:585-98.

5. Croom K. Ezetimibe for the treatment of primary hypercholesterolaemia. Drug Context 2008;4:153-72.

6. Kiekens F, Eelen S, Verheyden L, Daems T, Martens J, Van Den Mooter G. Use of ordered mesoporous silica to enhance the oral bioavailability of ezetimibe in dogs. J Pharm Sci 2012;101:1136-44.

7. Taupitz T, Dressman JB, Klein S. New formulation approaches to improve solubility and drug release from fixed dose combinations: case examples pioglitazone/glimepiride and ezetimibe/simvastatin. EurJ Pharm Biopharm 2013;84:208-18.

8. Mansouri M, Pouretedal HR, Vosoughi V. Preparation and characterization of ibuprofen nanoparticles by using solvent/antisolvent precipitation. Open Conf Proc J 2011;2:88-94.

9. George S, Vasudevan D. Studies on the preparation, characterization, and solubility of 2-HP- $\beta$-cyclodextrin-meclizine $\mathrm{HCl}$ inclusion complexes. J Young Pharm 2012;4:220-7.

10. Nicolescu C, Arama C, Monciu C. Preparation and characterization of inclusion complexes between repaglinide and $\beta$-cyclodextrin, 2 -hydroxypropyl- $\beta$-cyclodextrin and randomly methylated $\beta$-cyclodextrin. Farmacia 2010;58:78-83.

11. Ghorab MM, Babiker ME. Formulation and in vitro evaluation of isotritinoin tablets. J Chem Pharm Res 2012;4:2817-31.

12. Zhang D, Tan T, Gao L, Zhao W, Wang P. Preparation of azithromycin nanosuspensions by high pressure homogenization and its physicochemical characteristics studies. Drug Dev Ind Pharm 2007;33:569-75.

13. Kesisoglou F, Panmai S, Wu Y. Nanosizing - oral formulation development and biopharmaceutical evaluation. Adv Drug Deliv Rev 2007;59:631-44.

14. Bandi N, Wei W, Roberts CB, Kotra LP, Kompella UB. Preparation of budesonide- and indomethacin-hydroxypropyl-beta-cyclodextrin (HPBCD) complexes using a single-step, organic-solvent-free supercritical fluid process. Eur J Pharm Sci 2004;23:159-68.

15. Kosoglou T, Statkevich P, Johnson-Levonas AO, Paolini JF, Bergman AJ, Alton KB. Ezetimibe: a review of its metabolism, pharmacokinetics and drug interactions. Clin Pharmacokinet 2005;44:467-94.

16. Bali V, Ali M, Ali J. Nanocarrier for the enhanced bioavailability of a cardiovascular agent: in vitro, pharmacodynamic, pharmacokinetic and stability assessment. Int J Pharm 2011;403:46-5.

17. Bali V, Ali M, Ali J. Study of surfactant combinations and development of a novel nanoemulsion for minimising variations in bioavailability of ezetimibe. Colloids Surf B Biointerfaces 2010;76:410-20.

18. Dixit RP, Nagarsenker MS. Self-nanoemulsifying granules of ezetimibe: design, optimization and evaluation. Eur J Pharm Sci 2008;35:183-92.

19. Bandyopadhyay S, Katare OP, Singh B. Optimized self nano-emulsifying systems of ezetimibe with enhanced bioavailability potential using long chain and medium chain triglycerides. Colloids Surf B Biointerfaces 2012;100:50-61.

20. Gulsun T, Gursoy RN, Oner L. Design and characterization of nanocrystal formulations containing ezetimibe. Chem Pharm Bull (Tokyo) 2011;59:41-5.

21. Parmar KR, Shah SR, Sheth NR. Studies in dissolution enhancement of ezetimibe by solid dispersions in combination with a surfactant adsorbent. Dissolution Technol 2011;55-61. 